L. Hetey, J. Campbell, R. Vignjevic (2015), "Advisory system development for reliable FEM modelling in aerospace", Aircraft Engineering and Aerospace Technology, Volume 87, Issue 1, pp. 11-18. https://doi.org/10.1108/AEAT-04-2013-0067

\title{
Advisory system development for reliable FEM modelling in
}

\section{aerospace}

\begin{abstract}
Purpose - Today, there is no widely accepted tool for finite element (FE) idealisation error control. This paper describes the development of an advisory system that helps building sound FE models from CAD data, with actual uncertainty levels expressed by error values in percent.
\end{abstract}

Design/methodology/approach - The goal is to provide a computer-aided engineering (CAE) environment which assists the FE modelling phase. A demonstration program has been developed that leads the user through a step by step process and helps to detect idealisation errors. Uncertainties are identified and analysed following the procedure. An example illustrates the methodology on the collapse analysis of aerospace stiffened panels.

Findings - The design shows how a knowledge-based system can be used to aid a safe virtual product development.

Research limitations/implications - The extension of current CAE environments is difficult as the programs do not provide sufficient flexibility, changeability and FE solver independence. New developments can take the presented concept as a starting point.

Practical implications - The application of error control strategies increases the FE modelling fidelity and can prevent incorrect design decisions. The practical conversion of FE idealisation support depends on the ambitions of CAE software providers.

Originality/value - This research shows how a previously paper-and-pencil based error control procedure can be transformed to an easy-to-use tool in modern software.

Keywords - Advisory system, Expert system, FEM, Modelling, Idealisation, Error control

Paper type - Research paper 


\section{Introduction}

Today's structural engineering tasks are increasingly more complex and requirements to minimise weight and maximise performance are standard. This is especially important for new developments in the aeronautical industry. The FE method is thereby the most frequently applied calculation method. By dividing the whole structure into many small elements, a very good problem idealisation can be achieved. Each finite element is mathematically described with basic equations and contributes to the global solution by interacting with neighbouring elements.

Figure 1 SAFESA (SAFE Structural Analysis) as part of the CAE idealisation in the certification process

\section{CAE environment}

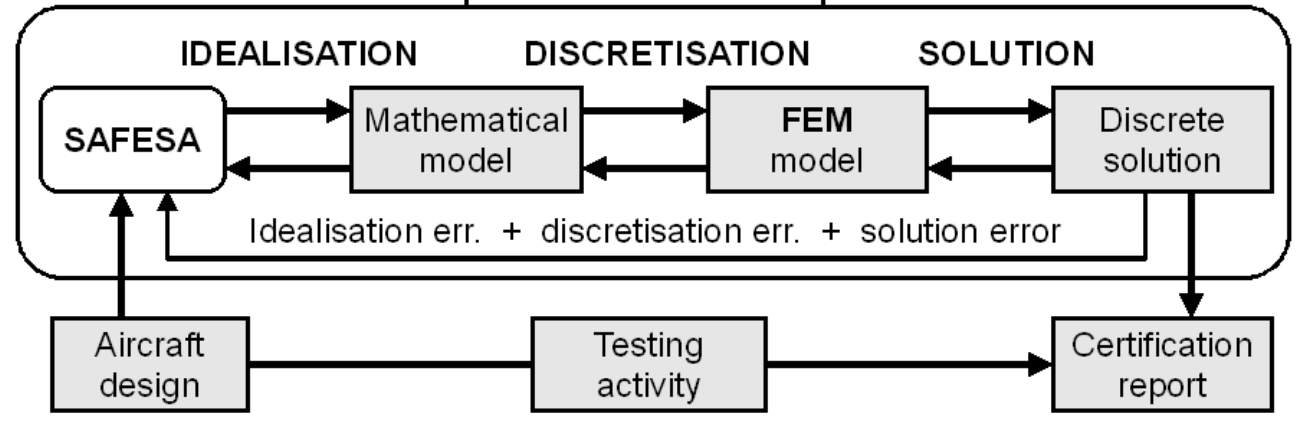

The FE idealisation process represents the different stages of converting a real-world structural design into an idealised model, Fig. 1. Within this process the analyst is required to make a series of assumptions, generally simplifications, which contribute to the error in the final analysis. Hereby an advisory system can help to improve the model reliability. The purpose is to provide a rigorous process for identifying these assumptions and controlling the resultant error. In the context of virtual product design and certification, the motivation is to reduce expensive testing with reliable simulation techniques.

This paper presents the implementation of an advisory system which extends the SAFESA (Symons and Morris, 1997) error control procedure to complex non-linear analysis cases. The idea is to demonstrate a concept that can be incorporated into CAE programs (such as CATIA, I-DEAS, MSC-PATRAN, ABAQUS/CAE) used by the industry to design new products. The advisory system motivates the user to justify every idealisation decision by asking to define a confidence level. If a decision cannot be made with certainty it needs to be analysed in detail.

The article is structured as follows: in the next section the error control procedure is outlined. Then related literature is reviewed and the motivation for this research explained. The subsequent sections survey current CAE tools and describe the advisory system implementation together with an aerospace modelling example. Finally, the program is evaluated and conclusions presented. 


\section{Idealisation error control procedure}

SAFESA is a procedure for formally controlling idealisation errors of linear static FE analysis. The input is a description of the real world problem, usually provided with computer-aided design (CAD) geometries, drawings, material properties etc. The output is a model ready for meshing. A flagging technology is applied to determine that the errors at a specific step have not been adequately treated and must be studied at a later stage. Information is fed from one step to the following in a sequential way and can involve analysis loops (Campbell et al., 2012):

- Step 1: Definition of geometry boundaries, boundary conditions and loading actions. Error bounds are estimated and all ambiguity is flagged out for later examination.

- Step 2: Definition of load paths and geometry simplification. Unnecessary structural details such as bolt holes or curved corners can be omitted.

- Step 3: Structural breakdown into features and primitives. A feature represents a recognisable entity, which exhibits coherent structural properties. A primitive is part of a feature and is the smallest possible fragmentation. The main idea behind this step is the study of feature interconnections, and the decomposition of a big problem into smaller ones.

- Step 4 \& 5: Iterating the first two steps at the feature and primitive level.

- Step 6: Evaluation of the performed analyses so far. Either error bounds can be directly assessed or supplementary testing (Step 7) is required.

- Step 7: Experimental testing. If the presumptions were unsuitable, they must be revised using the test results.

SAFESA was successfully applied in safety critical analysis cases. The first published application was the replacement of a window in a Jetstream aircraft by a metal sheet to place a radar aperture (Vignjevic et al., 1998). How far the methodology can be extended to more complex problems is the focus of current investigation (Morris, 2008; Hetey, 2009). The applicability for non-linear failure studies was investigated with the ultimate load analyses of aerospace stiffened panels (Campbell et al., 2012; Hetey et al., 2013), as described later in this paper.

\section{Literature on FE modelling support}

This research is related to Artificial Intelligence (AI) at the interface of CAD and FE structural analysis. Programs which help the decision making may be called Consultation System, Expert System (ES), Knowledge-Based System (KBS), Knowledge Based Engineering (KBE) tool or Advisory System, depending on the context, the related industry or the period of appearance (La Rocca, 2012). This field had several periods with high expectations followed with reduced interest and funding, see Sriram (2006) and Tomiyama (2007). Today, the term Advisory System may be a sensible choice for new developments in order not to remind of the disappointment of a specific expert system or technology. 
Early publications on advisory systems for aircraft structural design, such as Bennett et al. (1978), Taig (1986), Gregory and Shephard (1987), and Cagan and Genberg (1987), date back some decades. The programs used much less powerful computers without efficient visualisation facilities, however their basic ideas can still be applied today. Most of these systems work as an interactive consultation and provide qualitative suggestions. The FE models at that time were smaller and simplified the investigated structures, e.g. the analysis type was usually linear-static.

More recent developments focus on parametric modelling, automation and optimisation strategies. For this study the progress on reliable FE modelling support is of interest. Li and Qiao (2003) developed strategies for knowledge accumulation and decision support for the modelling of fuselage frames; especially the sub-structure connection modelling, selection of FEM element types and mesh refinement. Ledermann et al. (2005) report on parametric CAE methods in the pre-design of aircraft structures. Structures were split into parts and assemblies, where assemblies are built up of several parts. This allows a detailed analysis of the sub-component interconnections and a reliable modelling of boundary conditions. Modelling advice is derived from a database of parameters or can be calculated using heuristics. Dolšak and Novak (2011) developed intelligent decision support for structural design analysis, as selection of finite elements, mesh design and interpretation of results. The authors express the need for advisory systems to support the initial model idealisation, i.e. material modelling, contact definition, geometrical simplification and boundary conditions.

The CAD to FEM coupling is very important in the idealisation process. Chapman and Pinfold (1999) and Pinfold and Chapman (2001) discuss the limitations of the design engineering process. CAD and FEM do not use the same model representation hence the transformation requires major effort. But the design of new products is iterative and the result of negotiations, clarifications and evaluations. Every time a new FE model is set up its correctness has to be checked. The authors propose an advisor system that assists this process and checks important modelling decisions. Nawijn et al (2006) describe KBE methods for the CAD to FEM transformation. Support on structural segmentation and the selection of sub-component boundary conditions is provided as well as guidance on choosing material properties and FE element types. The functionality is implemented with an extension of MSC.Patran and then applied for the design of wings.

Several CAE programs offer an integrated knowledge handling interface. ICAD was the first commercially successful KBS for mechanical engineering. Boeing and Airbus used ICAD extensively to develop various components in the past decades. Cooper et al. (2001) and Morris et al. (2004) describe the successful use of ICAD for modelling aircraft structures. CATIA is the most widely applied design program in the aerospace industry today. Lendermann et al. (2005) discuss ideas for parametric modelling and how the KBE functionality of CATIA can be extended. Choi et al. (2007) investigated the transformation of geometrical data from CATIA to the FE solver Nastran, for the design of stiffened panels. 


\section{Motivation}

In spite of the large number of publications the commercial market for CAE decision support tools is limited. ICAD was bought by Dassault in 2002 and its maintenance discontinued a couple of years later (La Rocca, 2012). According to Mackerle (2002), in 2002 there was no commercial expert system available for reliable FEM, and the author was sceptical if any would appear in the near future. Reasons for the low commercial success comprise:

- high costs of software licences and required hardware (at their time) (La Rocca, 2012),

- tools were limited with many constraints, and could only be used for certain specific problems (Dolšak and Novak, 2011),

- lack of application examples (Verhagen et al., 2012),

- little connection or lack of integration with existing CAD systems (Tomiyama, 2007), and

- $\quad$ successful programs stayed hidden in laboratories of companies due to contractual reasons (Sriram, 2006).

Computing technology has evolved considerably since the development of the first advisory systems (Cooper and La Rocca, 2007). Early systems were running on very expensive LISP machines, which were much less efficient than today's desktop PCs. Modern technology improved the circumstances for advisory system developments, because:

- the cost of computing hardware has decreased enormously, and

- $\quad$ at the same time the computing power increased by several magnitudes, especially CAD visualisation and FEM solution speed,

- CAE environments offer KBE interfaces (CATIA, I-DEAS),

- development of new programming languages (e.g. object-oriented like $\mathrm{C}_{++}$, Java) and implementation strategies (e.g. design pattern, databases, visualisation, GUls),

- unified CAD data exchange formats (STEP, IGES),

- $\quad$ better meta-data and modelling languages (XML, UML),

- improved geometry visualisation formats (VRML, X3D),

- information and data availability via the World Wide Web, as there are

- $\quad$ open source / free compilers, software and libraries (Qt, OpenGL, OpenCascade, LISP derivatives, CLIPS, etc.).

Demands on users of modern CAE tools are greater because of the increased program complexity. Several commercial packages are available; each having specific features and limitations, and data formats are often incompatible. At the same time engineers are better trained in using software than doing hand calculations. This leads to a mentality of blind belief in computer, and unrealistic analysis results may be the consequence. On the other hand it is 
more convenient to develop new systems nowadays. This allows the implementation of programs with freely available software on standard PCs.

\section{Modelling support of current CAE tools}

The first step in the development process was to survey implementation platforms (Hetey, 2009). Several CAE environments used in the aircraft industry were compared, Table 1. The two main criteria were the available assistance in reliable FE modelling and the program extensibility. Packages like CATIA and I-DEAS provide CAD drawing, FE pre- and post-processing and their own FE solver. Both programs offer a KBS engine. The second group of tested programs are pre-processors like MSC.Patran, ABAQUS/CAE and Hypermesh. Each of them provides a programming interface to extend the program functionality.

Table 1 Comparison of CAE modelling tools.

\begin{tabular}{cccccc}
\hline $\begin{array}{c}\text { CAE } \\
\text { Package }\end{array}$ & $\begin{array}{c}\text { Idealisation } \\
\text { error control }\end{array}$ & $\begin{array}{c}\text { KBE } \\
\text { engine }\end{array}$ & $\begin{array}{c}\text { Program } \\
\text { extensibility }\end{array}$ & $\begin{array}{c}\text { Integrated } \\
\text { FE solver }\end{array}$ & $\begin{array}{c}\text { Export to different } \\
\text { FE solvers }\end{array}$ \\
\hline CATIA & limited & yes & limited & yes & limited \\
I-DEAS & limited & yes & limited & yes & limited \\
MSC.Patran & limited & - & yes & - & yes \\
ABAQUS/CAE & - & - & limited & yes & limited \\
Hypermesh & - & - & limited & - & yes \\
\hline
\end{tabular}

Some programs have the facility to check for FEM errors, e.g. for node offsets when importing CAD data or if the aspect ratios of FE elements are within the design limits. Of special interest was the available guidance for setting up a non-standard FE model; here the ultimate load determination of stiffened panels. For instance can the superficial choice of boundary conditions introduce errors up to $15 \%$. "Built-in" support (in FE language the restraint: $0,0,0,0,0,0$ ) is only an idealisation. When compressing stiffened panels up to their failure load the support boundary will differ from this idealisation. This potential error source needs first of all to be identified, and then analysed e.g. with a sensitivity study on a sub-model. Other error sources are the choice of non-linear material parameters and the modelling of geometrical imperfections (ideally with measured values). Also the modelling of joints and their failure can influence the structural failure load by several percentages. Standard packages cannot provide this specialised knowledge. But it would be helpful if they offer a facility to archive and share this knowledge.

The investigation deduced that the system should not be developed within one of the reviewed environments. None of the tested tools provided sufficient flexibility, changeability and FE solver independence. It appeared adequate to develop "from scratch" and to incorporate an expert system shell. 


\section{Program design}

The general idea for the advisory system implementation was to use open source software and international standards where possible, Fig. 2. This philosophy is driven by a desire to develop a flexible tool that can easily be modified to work in a variety of computing environments.

Figure 2 Advisory system design

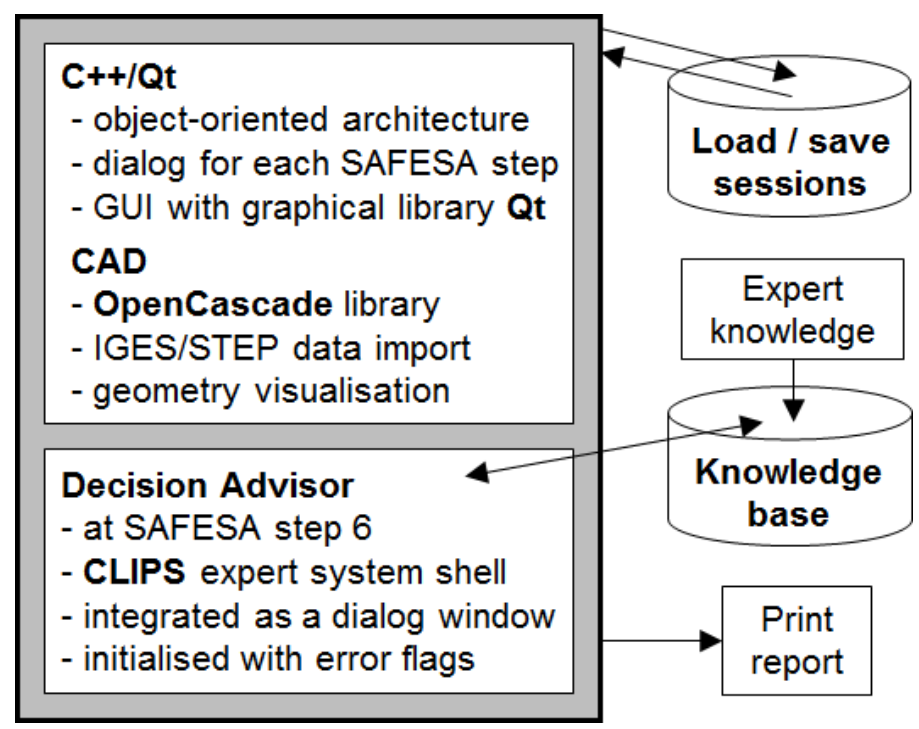

It was decided to program the demonstration software in $\mathrm{C}_{++}$(Microsoft Visual $\left.\mathrm{C}_{++}, 2010\right)$ with the help of the multiplatform libraries Qt (2011) and Open CASCADE (2012) on Windows-7. A decision advisor module was realised using the expert system shell CLIPS (2006). It seemed convenient to keep the CLIPS executable separate. This way, the advisor can be used independently or be replaced in the future with a more sophisticated KBE engine.

Figure 3 FE idealisation using the SAFESA advisory system

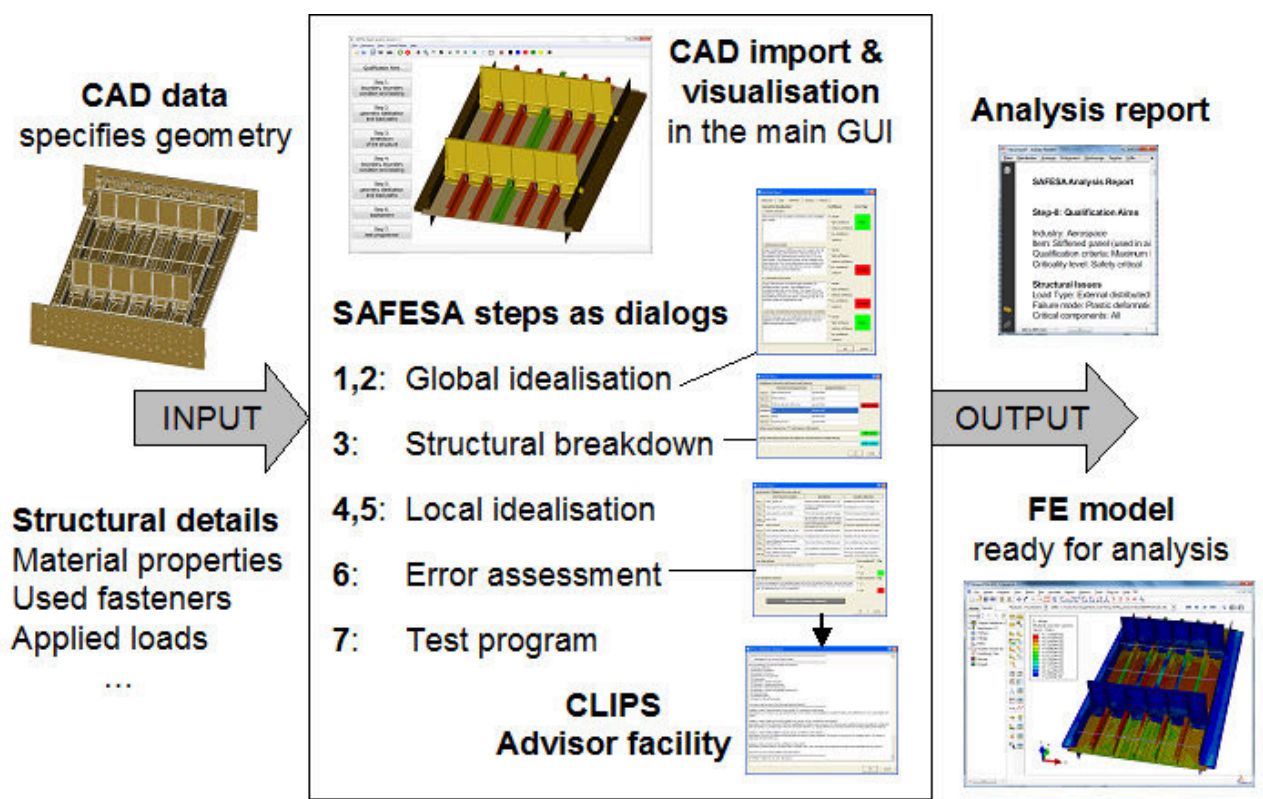


The system leads the users through the idealisation process, Fig. 3. To be more specific: the procedure forces the user to evaluate every modelling assumption, as each step is based on the output of the previous one. CAD data import and visualisation are performed in the program's main window. The SAFESA steps use separate dialogs. The decision advisor can be started in step-6 "Error assessment". For completed analyses, the program provides the facility to generate analysis reports. Each analysis session, including the idealisation audit trail and paths to CAD data, can be loaded and saved in text files. The documentation of successful analysis cases is one of the important program features. The advantage is that companies can archive the modelling experience, and do not lose knowledge due to a change in personnel.

\section{Beneficial programming libraries and applications}

Qt is a cross-platform application development framework that facilitated enormously the development of graphical user interfaces (GUI) in C++. For non-commercial projects the library can be used with its open-source version. Qt is developer friendly because it is well structured and contains an excellent documentation.

Open CASCADE (OCC) is a software development platform for three-dimensional CAE. The library includes components for solid modelling, visualisation and CAD data exchange. The basic types of geometrical entities are point, line, circle, plane and axis, which can be grouped into assemblies. When importing CAD geometry it is translated into an OCC shape. The shapes are saved internally via OCC pointers, which allow fast access and rendering.

The decision advisor facility uses the knowledge based system shell CLIPS (C Language Integrated Production System). This is a tool for representing a wide range of knowledge using rule based, object oriented and procedural programming. CLIPS was chosen as it is easy to use and provides a well documented programming interface.

\section{Knowledge acquisition interface}

The knowledge for the advisory consultancy is provided in textual format by the expert. This means the modelling expert should become familiar with the programming syntax (CLIPS, 2006) or an IT specialist translates the knowledge. Facts and rules are the basic elements of the syntax. A fact is a piece of knowledge expressed in pairs like (material steel) or (element_type shell). Program logic is created with the application of rules. In general, a rule is expressed in the form "IF something is true THEN do something".

The rules were formulated and organised in error categories (boundary conditions, load paths, etc.). Each category contains rules for general and for specific knowledge. A general knowledge rule provides global guidelines, as to wheter shells or solid elements are suitable. In a specific rule the user follows a decision tree by answering questions, e.g. how to 
model boundary conditions for stiffened panels. The provided level of uncertainty influences the system advice. The rules were formulated using the knowledge of previous analysis cases.

The advisory system is modular in design so that CLIPS can be replaced with another logic processing engine. The real difficulty is the accumulation and translation of analysis specific reasoning. Hereby, only engineers experienced in FE modelling can contribute.

\section{Application example}

This section describes the advisory system functionality. The demonstration case is the ultimate compressive load calculation of an Airbus stiffened panel, typical of those used in airplanes.

Figure 4 The advisory system showing imported CAD data

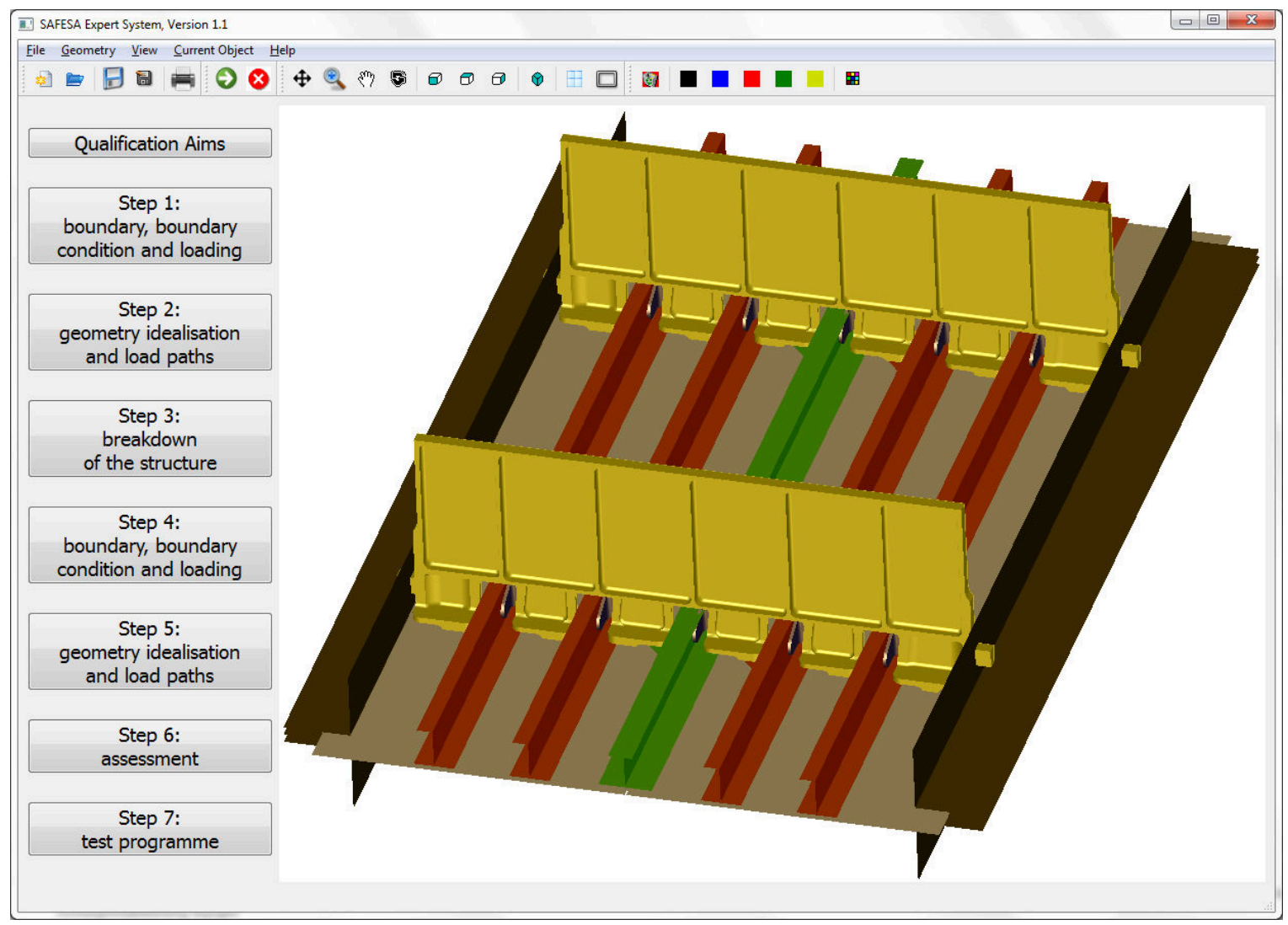

The first step of the modelling process is to import the geometry. The system can import the formats IGES and STEP, the two most commonly used formats for CAD data exchange in the industry. Fig. 4 shows the stiffened panel geometry visualised in the program's main window. Each feature can be assigned its own material and colour. Visualisation options are: fit the structure into the window, zoom, move or rotate. After loading the geometry, the SAFESA steps can be processed by clicking on the corresponding buttons, placed on the left side of the interface. 
Figure 5 Study of geometry idealisation in SAFESA step-2

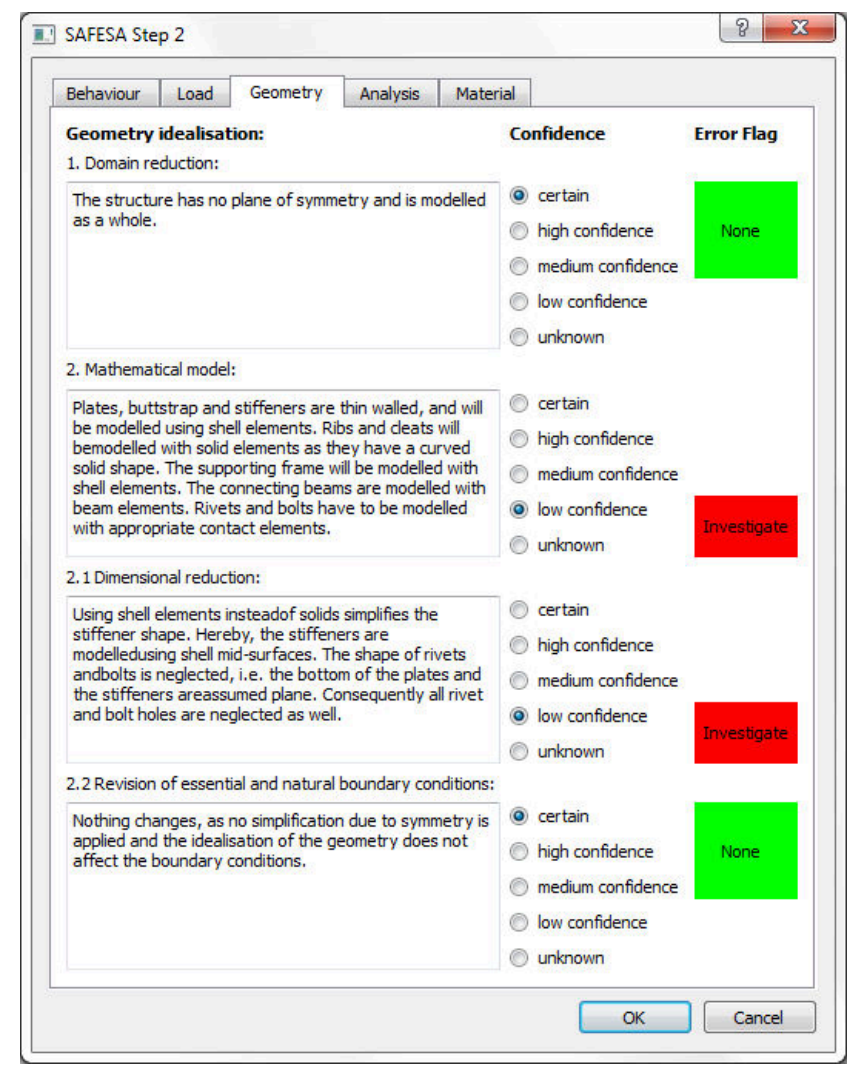

The impact of geometrical simplifications is analysed in SAFESA step-2. The corresponding dialog is separated into five tabulator views: behaviour, load, geometry, analysis and material, following the modelling logic, Fig. 5. Shown here is the geometry tabulator, where structural boundaries and dimensional reductions (e.g. solid versus shell elements) are defined. The user input is expressed in text. The confidence is specified by selecting a check-box from five categories: certain, high confidence, medium confidence, low confidence and unknown. Both "low confidence" and "unknown" will flag a potential error source, which is indicated with a red flag entitled "Investigate". 
Figure 6 List of flagged error sources at SAFESA step-6

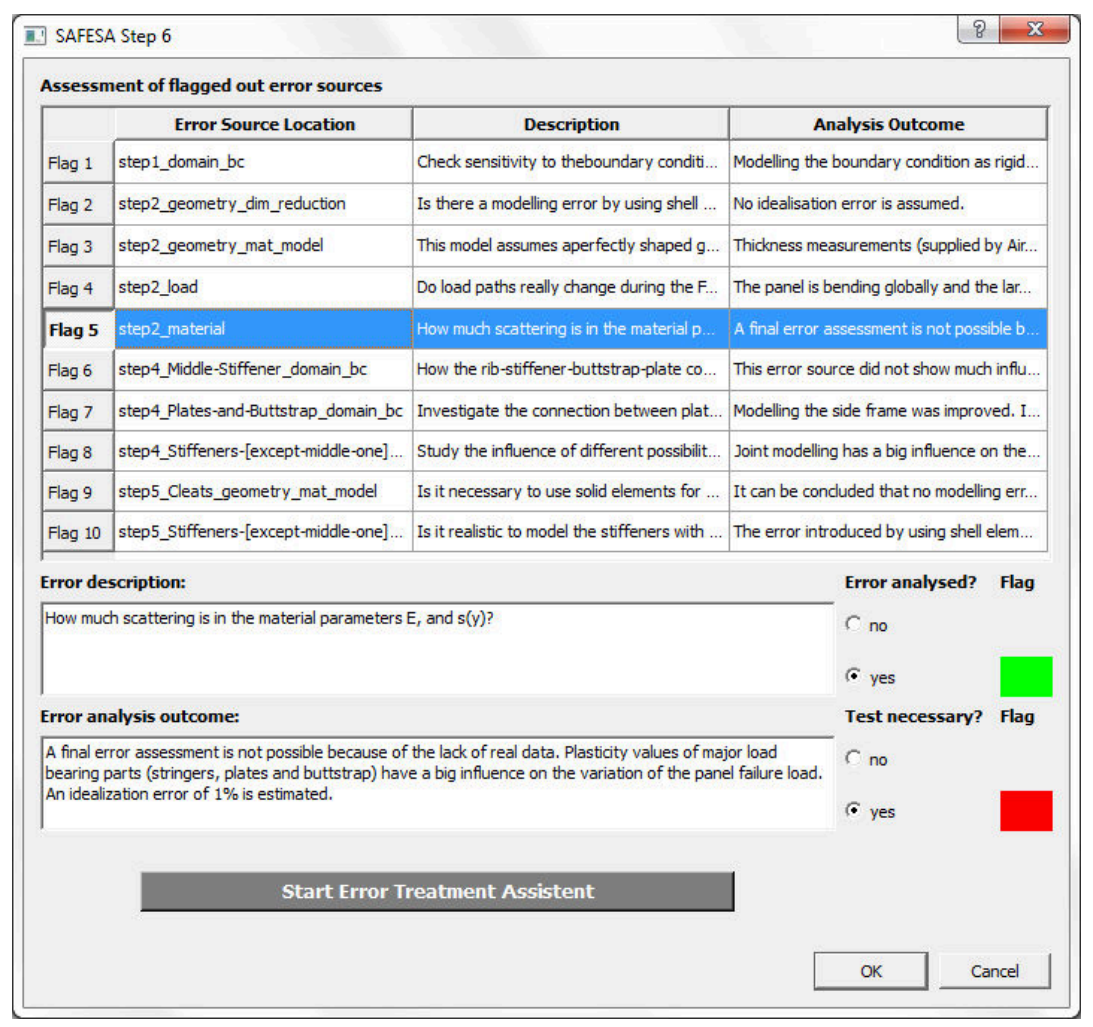

The modelling uncertainties are assessed in SAFESA step-6, Fig. 6. The upper part of the dialog lists the error sources which were flagged during the analysis. At this stage the "Error description", explaining the idealisation concern, is added. Once the error source is analysed and the provided "Error analysed?" check-box clicked, the upper flag will turn from red to green. The analysis outcome shall be provided in the input field "Error analysis outcome". The flag "Test necessary?" becomes red when additional testing has to be done. After completion, the flag can be switched to green, which indicates the completeness of the error treatment.

Step-6 is a very important stage in the process because the error sources have to be analysed and a remaining error value determined. Therefore, the decision advisor is applied at this stage. The CLIPS consultation is started by clicking the button "Start Error Treatment Assistant". The system analyses the error sources, their confidence level and possible interconnections with other uncertainties. 
Figure 7 Decision advisor consultation about boundary conditions

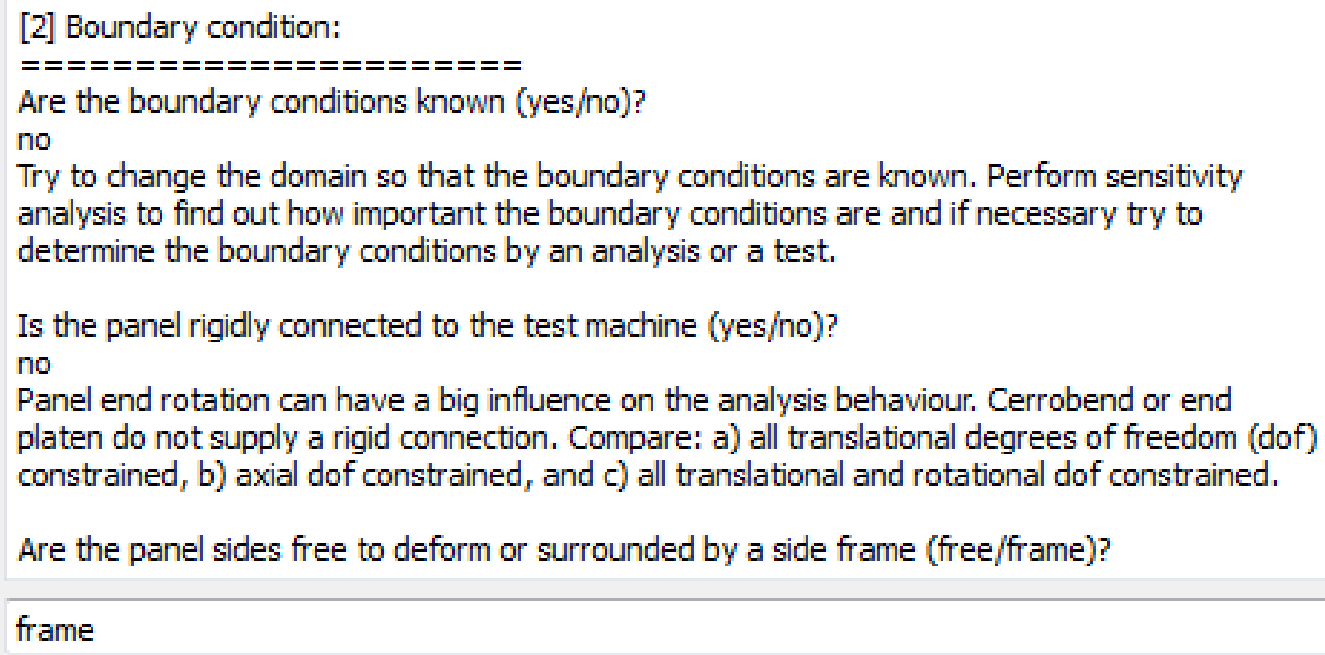

Fig. 7 illustrates a consultation with the decision advisor for the selection of appropriate boundary conditions. The system asks questions and leads the user through the decision process. Users can read the output in the text field and respond to the system using the input field at the bottom. In the example, advice is given depending on how the structure is placed in the test environment. At this stage an extension of the original SAFESA procedure was necessary, in order to cope with the non-linear error sources, e.g. modelling of joint failure, plastic material deformation or the realistic evolution of load paths during the simulation. The compiled knowledge in this demonstration is specific for the analysis of stiffened panels, but can be adapted to other application domains.

\section{Program testing and evaluation}

The advisory system was employed for two FE idealisation error analyses of stiffened panels. The immediate feedback influenced the final program structure. The first panel was thin-walled and showed highly non-linear deformation behaviour. The simulated collapse load differed less then $8 \%$ of the tested failure value (Campbell et al., 2012). The second panel was thick-walled and similar to those employed in airplanes. The results could be verified with industry supplied test data. The measured collapse load was 3.5\% lower than the one predicted with FE (Hetey et al., 2013). These are very good results and important for a safe design of future aircraft.

The program was evaluated by several senior structures engineers. The task was to repeat their last FE modelling, this time with the focus on idealisation error control. The user feedback was very positive because some previously "negligible" assumptions turned into real error sources, when analysed carefully. This happened mainly because the methodology forces every decision to be justified. Overall, the engineers involved during testing expressed an increased confidence in their FE model. 


\section{Conclusion}

The objective of this research was to derive a development strategy for an advisory system. The key requirement was to provide guidance on carrying out reliable FE analyses with audit trail functionality. A decision advisor, CAD data import and geometry visualisation were additional features. The implemented concept shows how a knowledge-based system can be used to aid the virtual product design. The program provides a prototype which can be extended or ported to other CAE platforms.

\section{Acknowledgments}

The work presented in this paper was financially supported by the project MUSCA ("non-linear static MUltiSCAle analysis of large aero-structures"), partially funded by the European Union under the Sixth Framework Programme (Project Reference 516115). The authors would like to thank the OpenCascade forum community, Prof. Alan Morris, Prof. Tetsuyo Tomiyama, Dr. Christian Sandor and Dirk Schumann for their support and the reviewers for their advice.

\section{References}

Bennett, J., Creary, L., Englemore, R. and Melosh, R. (1978), "SACON: A knowledge-based consultant for structural analysis", Technical Report, STAN-CS-78-699, Stanford University.

Cagan, J. and Genburg, V. (1987), "PLASHTRAN: An expert consultant on two-dimensional finite element modelling techniques", Engineering with Computers, Vol. 2, pp. 199-208.

Campbell, J., Hetey, L. and Vignjevic, R. (2012), "Non-linear idealisation error analysis of a metallic stiffened panel loaded in compression", Thin-Walled Structures, Vol. 54, pp. 44-53.

Chapman, C.B. and Pinfold, M. (1999), "Design engineering - a need to rethink the solution using knowledge based engineering", Knowledge-Based Systems, Vol. 12 No. 5-6, pp. 257-267.

Choi, J.-W., Kelly, D. and Raju, J. (2007), "A knowledge-based engineering tool to estimate cost and weight of composite aerospace structures at the conceptual stage of the design process", Aircraft Engineering and Aerospace Technology, Vol. 79 No. 5, pp. 459-468.

CLIPS 6.24 (2006), Public domain tool for building expert systems, available at: http://clipsrules.sourceforge.net (accessed April 2013).

Cooper, D. and La Rocca, G. (2007), "Knowledge-based techniques for developing engineering applications in the 21st century", Proceedings of the 7th AIAA Aviation Technology, Integration and Operations Conference, AIAA Belfast, Northern Ireland, 2007.

Cooper, S., Fan, I.-S. and Li, G. (2001), "Achieving competitive advantage through knowledge based engineering - a best practice guide", White Paper for the Department of Trade and Industry, Cranfield University, Cranfield.

Dolšak, B. and Novak, M. (2011), "Intelligent decision support for structural design analysis", Advanced Engineering Informatics, Vol. 25 No. 2, pp. 330-340.

Gregory, B.L. and Shephard, M.S. (1987), "The generation of airframe finite element models using an expert system", Engineering with Computers, Vol. 2 No. 2, pp. 65-77.

Hetey, L. (2009), “Idealisation error control for aerospace virtual structural testing”, Ph.D. thesis, Cranfield University, Cranfield. 
Hetey, L., Campbell, J. and Vignjevic, R. (2013), "Non-linear idealisation error analysis of an aerospace stiffened panel loaded in compression", Proceedings of the Institution of Mechanical Engineers, Part G: Journal of Aerospace Engineering: 0954410013497151, first published on August 12, 2013 as doi: 10.1177/0954410013497151.

La Rocca, G. (2012), "Knowledge based engineering: between Al and CAD, review of a language based technology to support engineering design", Advanced Engineering Informatics, Vol. 26 No. 2, pp. 159-179.

Ledermann, C., Hanske, C., Wenzel, J., Ermanni, P. and Kelm, R. (2005), "Associative parametric CAE methods in the aircraft pre-design", Aerospace Science and Technology, Vol. 9 No. 7, pp. 641-651.

Li, S. and Qiao, M. (2003), "A hybrid expert system for finite element modeling of fuselage frames", Expert Systems with Applications, Vol. 24 No. 1, pp. 87-93.

Mackerle, J. (2002), "FEM and BEM in the context of information retrieval", Computers and Structures, Vol. 80 No. 20 21, pp. 1595-1604.

Microsoft Visual C++ 2010 Express (2010), Public domain compiler, available at: http://www.microsoft.com/visualstudio/en-us/products/2010-editions/visual-cpp-express (accessed April 2013).

Morris, A., Arendsen, P., La Rocca, G., Laban, M., Voss, R. and Hönlinger, H. (2004), "MOB - a European project on multidisciplinary design optimisation", Proceedings of the 24th ICAS Congress, ICAS, Yokohama, Japan, 2004.

Morris, A. (2008), "A practical guide to reliable finite element modelling", Wiley.

Nawijn, M., van Tooren, M.J.L., Berends, J.P.T.J. and Arendsen, P. (2006), "Automated finite element analysis in a knowledge based engineering environment", Proceedings of the 44th AIAA Aerospace Science Meeting and Exhibit, Reno, USA, 2006.

Open CASCADE 6.5.3 (2012), Software development platform, available at: http://www.opencascade.org (accessed April 2013).

Pinfold, M. and Chapman, C. (2001), "The application of KBE techniques to the FE model creation of an automotive body structure", Computers in Industry, Vol. 44 No. 1, pp. 1-10.

Qt 4.8.0 (2011), Cross platform application development platform, available at: http://qt.nokia.com (accessed April 2013).

Sriram, R.D. (2006), "Artificial intelligence in engineering: personal reflections", Advanced Engineering Informatics, Vol. 20 No. 1, pp. 3-5.

Symons, C. and Morris, A. (1997), "Analytic certification of airframes", Aircraft Engineering and Aerospace Technology, Vol. 69 No. 3, pp. 235-240.

Taig, I.C. (1986), "Expert aids to reliable use of finite element analysis", in Bathe, K.-J. and Owen, D.R.J. (Eds.), Reliability of Methods for Engineering Analysis, Pineridge Press, pp. 457-474.

Tomiyama, T. (2007), "Intelligent computer-aided design systems: past 20 years and future 20 years", Al EDAM: Artificial Intelligence for Engineering Design, Analysis and Manufacturing, Vol. 21 No. 1, pp. 27-29.

Verhagen, W.J.C., Bermell-Garcia, P., van Dijk, R.E.C. and Curran, R. (2012), "A critical review of knowledge-based engineering: an identification of research challenges", Advanced Engineering Informatics, Vol. 26 No. 1, pp. 5-15.

Vignjevic, R., Morris, A.J. and Belagundu, A.D. (1998), "Towards high fidelity finite element analysis", Advances in Engineering Software, Vol. 29 No. 7-9, pp. 655-665. 


\section{Cranfield University}

2015-01-05

\section{Advisory system development for reliable FEM modelling in aerospace}

Hetey, Laszlo

Emerald

Hetey L, Campbell J, Vignjevic R. (2015) Advisory system development for reliable FEM modelling in aerospace. Aircraft Engineering and Aerospace Technology, Volume 87, Issue 1, January 2015, pp. 1-18

https://doi.org/10.1108/AEAT-04-2013-0067

Downloaded from Cranfield Library Services E-Repository 